\title{
Adverse Event Characteristics
}

National Cancer Institute

\section{Source}

National Cancer Institute. Adverse Event Characteristics. NCI Thesaurus. Code C53251.

The distinguishing attributes and qualities of an adverse event. 